\title{
REVIEW
}

\section{Alternative uses of dermoscopy in general dermatology}

\author{
Eleni Papakonstantinou ${ }^{1 *}$, Ulrike Raap ${ }^{1,2}$ \\ ${ }^{1}$ Department of Dermatology and Allergy, Hannover Medical School, Germany \\ ${ }^{2}$ Department of Dermatology and Allergy, Medical Center Oldenburg, Germany
}

\begin{abstract}
Over the last few years, dermoscopy has been shown to be an effective diagnostic tool in assisting the noninvasive diagnosis not only of skin tumors but also of various general dermatological disorders. The use of dermoscopy has offered a new morphological dimension supporting the diagnosis and clinical follow-up of several dermatological conditions. Within the last years, dermoscopy is gaining ground in general dermatology as well. Among the large spectrum of skin infections, specific dermoscopic patterns have been described for common skin infections such as viral warts, molluscum contagiosum, scabies, and pediculosis, as well as for tinea nigra, tungiasis, and cutaneous larva migrans. Also, common skin disorders such as psoriasis, pityriasis rosea or lichen planus show characteristic dermoscopic patterns. The aim of this article is to provide an overview of the use of dermoscopy in general dermatology by analyzing the dermoscopic differential diagnosis of relatively common dermatological disorders grouped according to their clinical characteristics.
\end{abstract}

Keywords: Dermoscopy; general dermatology; inflammoscopy; entomodermoscopy

Citation: Papakonstantinou E and Raap U. Alternative uses of dermoscopy in general dermatology. J Surg Dermatol 2017; 2(2): 67-74; http://dx.doi.org/10.18282/jsd. v2.i2.122.

*Correspondence to: Eleni Papakonstantinou, Department of Dermatology and Allergy, Hannover Medical School, Carl-Neuberg-Str. 1, 30625 Hannover, Germany, Papakonstantinou.Eleni@mh-hannover.de.

Received: $13^{\text {th }}$ December 2016; Accepted: $15^{\text {th }}$ December 2016; Published Online: $15^{\text {th }}$ February 2017

\section{Introduction}

Dermoscopy is a non-invasive technique that allows for a rapid and magnified in vivo observation of the skin surface with the visualization of morphologic features which are normally invisible to the naked eye ${ }^{[1]}$. Dermoscopy is performed using various manual devices called dermoscopes whose function is based on a magnifying glass which allows a 10 -fold magnification. When combined with a contact medium (for example, immersion oil), dermoscope enables the visualization of the structures of the basal layers of epidermis and upper dermis $^{[2]}$. New generations of commercially available hand-held dermoscopes apply polarized light and do not require a contact medium and thus preclude a direct contact between the optical lens and the $\operatorname{skin}^{[3]}$. These devices can be therefore applied without the risk of possible transfection because this risk is considered to be $\operatorname{low}^{[4,5]}$.

These hand-held dermoscopes fit into the pocket and can be easily used in the daily practice. In recent years, the use of dermoscopy in general dermatology is increasing, including in the evaluation of inflammatory and infectious diseases, parasitoses, hair abnormalities and a great variety of other dermatological conditions far removed from the classical fields of application in the differential diagnosis of pigmented skin lesions ${ }^{[6]}$.

Such skin infections affect thousands of people worldwide, and a delayed or incorrect diagnosis may have a negative impact not only on the prognosis of the patient but also on the healthcare system. Till recently, the diagnosis of skin infections has been based on spe-

Copyright (c) 2017 Papakonstantinou E and Raap U. This is an Open Access article distributed under the terms of the Creative Commons Attribution-NonCommercial 4.0 International License (http://creativecommons.org/licenses/by-nc/4.0/), permitting all non-commercial use, distribution, and reproduction in any medium, provided the original work is properly cited. 
cific clinical features in combination with histopathological and laboratory findings. Among the large spectrum of skin infections, specific dermoscopic patterns have been described for several skin infections and disorders.

Dermoscopy demonstrates an important diagnostic tool helpful in the differential diagnosis, prognostic evaluation, and treatment monitoring. Depending on the field applied, dermoscopy is divided into inflammoscopy and entomodermoscopy.

In this review article, we focus on the role of dermoscopy in the diagnostics and therapeutic follow-up of several common skin disorders and infections encountered in clinical daily practice.

\section{Inflammoscopy}

The term inflammoscopy refers to the application of dermoscopy on inflammatory skin disorders. Several inflammatory skin diseases display characteristic dermoscopic patterns which not only help the diagnosis but also the differentiation from other clinically similar diseases. Furthermore, dermoscopy represents a useful tool for the evaluation of therapeutical outcome and also for monitoring the disease by the recognition of structural or vascular changes before they can clinically be observed.

Since inflammatory and infectious diseases usually affect multiple or large areas of the body, it is essential to perform a full body examination of the patient. The dermoscopic examination follows the clinical one and is based on the evaluation of specific vascular pattern, its architectural arrangement within the lesion, and the presence or absence of additional characteristic dermoscopic features. Following these steps will help the clinician establish an accurate final diagnosis. In this first section, we are going to discuss the role of dermoscopy in common skin disorders such as psoriasis, lichen planus, and pityriasis rosea.

\section{Psoriasis}

Psoriasis commonly appears with erythematous plaques, which are characterized by surface scaling of various severities (Figure 1A). Dermoscopy of plaque psoriasis reveals the characteristic of white scales and homogenously distributed dotted vessels on a slightly red ground. Usually, hyperkeratotic plaques have to be slightly removed in order to identify the underlying vascular structures (Figure 1B). The evaluation of vascular pattern is relevant in order to investigate its pathogenesis as well as to enhance the diagnosis of psoriasis ${ }^{[7]}$. Vessels appear dermoscopically as small red dots that are symmetrical and regularly distributed throughout the psoriasis lesions, in which it characterizes and specifies the diagnosis of psoriasis ${ }^{[8]}$. Although relatively uncommon, "red globular rings" have also been described as a dermoscopic sub-pattern of plaque-type psoriasis, but are rather rare ${ }^{[9]}$.

Using higher magnifications $(100 \mathrm{X}-400 \mathrm{X})$, the psoriatic vessels appear as convoluted loops, mirroring the underlying histopathological finding of curly capillaries within the dermal papillae, which are associated with the psoriasiform epidermal hyperplasia ${ }^{[10]}$.

Dermoscopy can distinguish psoriasis from other skin disorders appearing with scaling plaques such as actinic keratosis, squamous cell carcinoma or superficial basal cell carcinoma ${ }^{[11-13]}$. Psoriasis can also be differentiated from dermatitis subtypes through the recognition of characteristic yellow scale/crusts and the patchy vascular distribution within the lesion ${ }^{[8]}$.

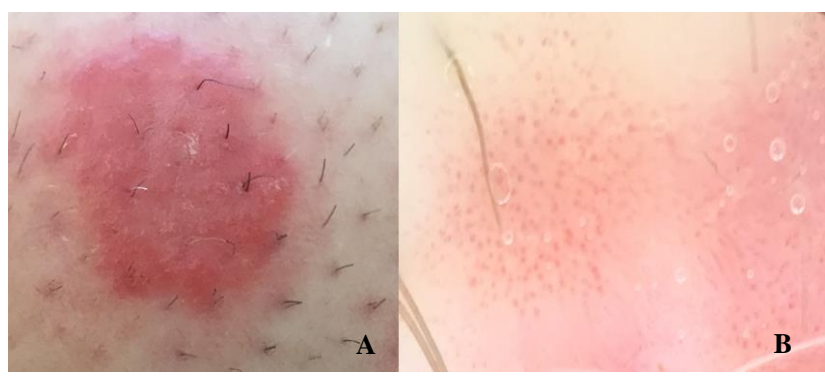

Figure 1. (A) Clinical manifestation of a psoriasis plaque after removing of the scaling surface. (B) Dermoscopic examination of this plaque psoriasis reveals homogenously distributed red dotted vessels on a slightly red ground.

\section{Lichen planus}

The dermoscopic hallmark of lichen planus is the Wickham striae, which suggests that it is highly sensitive and a specific criterion for the diagnosis of lichen planus ${ }^{[8,14]}$. Wickham striae can appear as pearly-whitish structures, and rarely as yellow or blue-white ones. Its morphological patterns vary from reticular, which is the most common, to linear, annular, round or displaying a "radial streaming", "leaf venation" or "starry sky" patterns ${ }^{[8,14-17]}$. The Wickham striae are commonly surrounded by dotted, globular, and/or linear vessels, mainly localized at the periphery of the lesion.

Active lesions of lichen planus may dermoscopically reveal violet, reddish, pink, brown or yellow background, with white or yellow dots and some pigmented reticular or cloud-like structures ${ }^{[8,16,17]}$. In older lesions of lichen planus, pigmented structures may appear, thus making the vessel pattern less obvious ${ }^{[15]}$. Comedo-like structures filled with corneal content may also appear ${ }^{[18]}$. Lichen planus can be dermoscopically diagnosed by 
revealing the pathognomonic Wickham striae ${ }^{[8,19]}$. Besides the diagnostic role of dermoscopy, useful information for the monitoring of pigmented lichen planus can be obtained. Therefore, lesions of pigmented lichen planus developing resistant grey-blue-brown granules seem to persist longer in comparison to lesions with brownish structureless areas ${ }^{[20]}$.

\section{Pityriasis rosea}

Pityriasis rosea clinically manifests with a round-to-oval irregularly sized erythematous patches following the Blaschko's lines and often revealing a scaling surface (Figure 2A). The dermoscopic examination reveals a characteristic of "collarette scaling" resistance of a peripheral whitish scaling and dotted vessels which are lacking of a regular pattern and are irregularly or focally distributed, which make them different from that of psoriasis (Figure 2B). Additionally, diffuse or localized yellowish-orange structureless areas may be dermoscopically apparent ${ }^{[8,21]}$. Pityriasis rosea can thus be easily differentiated from other dermatitis subtypes which manifest with yellow crusts but without the typical collarette of white scales.

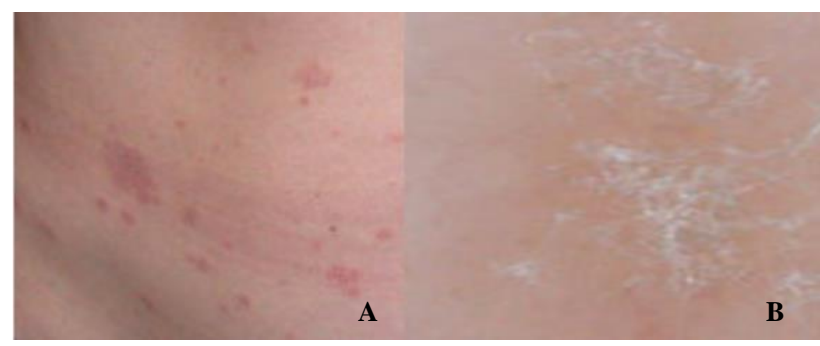

Figure 2. (A) Clinical manifestation of pityriasis rosea with round to oval irregularly sized erythematous patches with a light scaling surface which follow the Blaschko's lines. (B) Dermoscopic examination of pityriasis rosea reveals a "collarette scaling" characteristic resistance of a peripheral whitish scaling. The dotted vessels cannot be recognized in this figure.

\section{Dermatitis}

The term dermatitis covers a wide spectrum of diseases such as nummular eczema, allergic contact dermatitis or atopic dermatitis. All subtypes of dermatitis are primarily characterized by dotted vessels in a patchy distribution and yellow serocrusts or scaling ${ }^{[2,23]}$. Depending on the stage of the disease, eczematous dermatitis displays some common characteristics: acute exudative lesions are characterized by yellow scale/crusts ("yellow clod sign") while chronic, lichenified lesions appear mainly with patchily distributed dotted vessels within the lesion $^{[24-26]}$.

\section{Mycosis fungoides}

The typical dermatoscopic features of mycosis fungoides consist of short curved-linear vessels with orangeyellowish patchy areas. Furthermore, fine white scaling and dotted vessels can also be seen ${ }^{[27]}$. The diagnosis of mycosis fungoides during the early stage is enhanced by the presence of different vascular patterns. In particular, lesions of mycosis fungoides display characteristic thin linear vessels either alone or in combination with red dots, which are overlooked in other dermatitis subtypes ${ }^{[27]}$.

In the above-mentioned dermatological conditions, dermoscopy can also be useful in the evaluation of therapeutic outcome and the monitoring of the disease. Long-term topical therapy with steroids can cause skin atrophy and teleangiectasies, which are visible using dermoscope in early stages and before they become clinically apparent ${ }^{[28]}$. Furthermore, dermoscopy can be a potentially useful tool for the monitoring of the disease, besides evaluation and prediction of the disease reoccurrence based on vascular alterations after systemic therapy ${ }^{[29,30]}$.

\section{Infectious skin diseases-Entomoder- moscopy}

Entomodermoscopy represents another chapter of dermoscopy and points out the role of this technique as a link between dermatology and entomology for daily clinical diagnostics practice.

\section{Viral skin infections}

\section{Viral warts}

Cutaneous warts are common benign epidermal proliferations caused by various strains of human papilloma virus. Depending on their location, warts are typically classified as verruca vulgaris (common warts, typically involving human papilloma virus strains 2 or 7), plane warts (strains 3 or 10), verruca plantaris or palmaris (plantar or palmar warts, strains 1, 2, and 4) and condyloma acuminata (anogenital warts, strains include $6,11,42,43$, and 44).

Although their diagnosis can be mostly clinically accessed, clinicians may face difficulties with clinical features that overlap with skin lesions or mimic other skin diseases. Therefore, dermoscopy can be again a useful diagnostic tool for the establishment of the diagnosis. 


\section{Verruca vulgaris}

Verruca vulgaris is a keratotic papilloma of the epidermis that occurs most frequently in young people as a result of localized infection by human papilloma virus usually of types 2 or 7 . The dermoscopic hallmark of verruca vulgaris consists of large dotted vessels corresponding to multiple densely located papillae, with a red dot in the middle or a loop on white background, i.e. "whitish halo", which gives an appearance reminiscent of frogspawn. These dermoscopic features help the diagnosis of doubtful cases, e.g. irritated warts, or difficult localizations, e.g. subungual or periungual warts ${ }^{[31,32]}$.

\section{Plane warts}

Plane warts are flat-topped, skin-colored exophytic skin lesions that most commonly occur on the face and extremities of the patients and are caused by human papilloma virus usually of types 3 or 10 . The dermoscopic hallmark of plane warts is multiple small, regularly distributed red dots on a light brown background, which correspond to small vessels in the papillary dermis $^{[31,32]}$. Dotted vessels of plane warts are in contrast to those of verruca vulgaris smaller and not associated with hemorrhages.

\section{Verruca palmaris or plantaris}

Verruca plantaris or palmaris are viral warts located on palms or soles of the patients and most of the time are commonly caused by strains 1,2 , and 4 of human papilloma viruses. In contrast to the previously mentioned types of palmoplantar warts, it lacks dotted or looped vessels. The dermoscopic typical feature is a verrucous, structureless, yellowish or brownish area with irregularly distributed red, brown or black dots or linear streaks due to hemorrhages. These are present predominantly on plantar areas due to the chronic pressure of the vessels on these locations. Dermoscopy can be very helpful when it comes to differential diagnosis of skin lesions on palmoplantar areas. Hyperkeratotic areas due to chronic friction lack vascular structures and can be easily recognized on these sites. When it comes to pigmented lesions on palmoplantar areas, acral lentiginous melanoma is the most common differential diagnosis.

This is a rather aggressive variant of melanoma and is often missed or very tardily diagnosed, resulting in a bad prognosis for the patient. Asymmetry of color and shape, atypical network, and presence of blue-white structures or veil are characteristics suspicious for acral melanoma. Furthermore, pigmentation on the ridges (parallel ridge pattern) is a highly specific dermoscopic pattern and raises the index of suspicion ${ }^{[33]}$. Besides the diagnostic impact, dermoscopy may also be useful in monitoring the treatment of viral warts. Disappearance of hemorrhages and vascular pattern is a sign of effective therapy and predicts a low risk of recurrence ${ }^{[34]}$.

\section{Anogenital warts}

Anogenital warts are exophytic papillomas typically found on the skin or mucous membranes of the anus and external genitalia, and are caused by a human papillomavirus usually of types $6,11,42,43$, and 44 .

In dermoscopy, anogenital warts are presented with a non-regular patchwork consisting of unaffected mucosal skin and areas with white reticular network ${ }^{[34]}$. Several morphologic patterns such as finger-like, knob-like or mosaic-like along with vascular structure shave have been described ${ }^{[35,36]}$. Typically, single red dots can often be seen corresponding to glomerular vessels ${ }^{[37]}$.

\section{Molluscum contagiosum}

Molluscum contagiosum is a common skin infection caused by a highly contagious poxvirus. The typical dermoscopic pattern of molluscum contagiosum consists of a central pore (umbilication) together with polylobular white-to-yellow amorphous structures, which are surrounded by reddish, linear or branched vessels ("corona"-like vessels) ${ }^{[38,39]}$. These characteristic features enable the differentiation of molluscum contagiosum from other types of skin lesions. Dermoscopy performed on molluscum contagiosum lesions seems to be superior to dermatological examination in the diagnosis of this skin disorder and helpful when it comes to pediatric patients $^{[40,41]}$.

\section{Fungal infections}

\section{Tinea nigra}

Tinea nigra is a superficial skin infection caused by Phaeoannellomyces werneckii, formerly called Cladosporium werneckii or Exophiala werneckii. Tinea nigra commonly affects children or young adults living in or traveling to tropical or subtropical areas of South and Central America, Asia or Africa, but has also been described in the United States and Europe. Clinically, it manifests mostly on the palms and soles as an irregular, brown-to-black macule and is often asymptomatic.

Since tinea nigra can mimic melanocytic lesions such as junctional nevi or acrolentiginous melanoma, the dermoscopic examination provides important evidence for 
the establishment of the right diagnosis. In contrast to acral melanocytic lesions, tinea nigra dermoscopically reveals superficial wispy brown strands or "pigmented spicules" that form a reticular-like patch. Furthermore, tinea nigra does not have any melanocytic pattern such as pigmentation to the furrows or ridges of the volar skin, which is typical in the case of nevi or melanomas ${ }^{[42]}$. It has been proved that the diagnosis of tinea nigra has been significantly improved by using dermoscopy ${ }^{[43]}$. The use of dermoscopy can also prevent unnecessary biopsies in critical cases which may become a difficult issue, especially in pediatric patients.

\section{Pityriasis versicolor}

Pityriasis versicolor is a common, superficial skin fungal infection caused by Malassezia species such as Malassezia globosa and Malassezia furfur, and is usually characterized by hypopigmented or hyperpigmented macules and patches on the chest and the back of the patients. Histopathologically, a characteristic finding of "spaghetti-and-meatball" formations, which demonstrate the presence of fungal forms, can be found. Since the clinical appearance of pityriasis versicolor may sometimes be confusing, dermoscopy can provide important information and contribute to the establishment of diagnosis. The typical dermoscopic findings of pityriasis versicolor are multiple-pigmented stripes with fine scales in a network-like distribution ${ }^{[44]}$. Therefore, it can lighten the load of diagnosis and prevent unnecessary invasive procedures such as biopsy.

\section{Parasitoses}

\section{Scabies}

Scabies is a highly contagious itchy skin disorder caused by a tiny burrowing mite called Sarcoptes scabiei. The presence of the mite leads to intense itching in the area of its burrows. Dermoscopy represents a useful and effective diagnostic tool for scabies with diagnostic sensitivity values comparable to the ex vivo microscopic examination, which takes less time and effort ${ }^{[45]}$. Furthermore, it enhances the diagnostic skills for making treatment decisions even among inexperienced clinicians.

The typical dermoscopic feature of scabies consists of a small dark-brown triangular structure at the end of whitish structureless and characteristic curved or wavy lines, giving an appearance reminiscent of a delta-wing jet with contrail. The brown triangle corresponds to the pigmented anterior part of the mite (the head and the two anterior pairs of legs), while the posterior part which corresponds to the abdomen and legs appear translu- cent and is subsequently not observed by dermoscopy ${ }^{[46,47]}$. Interestingly, dermoscopy is known to be useful in the therapeutic follow-up of scabies as well ${ }^{[48]}$.

The disappearance of all existing lesions, no formation of new lesions, and no detection of any dermoscopic jet with contrail features on the entire skin lesions predict a successful treatment. If skin lesions persist one or two weeks after an antiscabietic therapy, a dermoscopic evaluation for a jet with condensation trails or the hang glider is indicated. With the presence of these findings, which are signs of an active infection, repeated usage of the antiscabietic therapy is essential; otherwise an anti-inflammatory therapy can be $\operatorname{started}^{[49]}$. It must be noted that the itch, which represents the most bothersome clinical symptom of scabies infection, may persist for 4-6 weeks after successful eradication of the scabies mite ${ }^{[50,51]}$.

\section{Pediculosis capitis}

The diagnosis of pediculosis capitis is typically clinically accessed based on the identification of either adult lice or viable nits through close-up examination. In pediculosis capitis, dermoscopy ensures a more detailed evaluation of both mites and eggs, particularly in the presence of post-treatment residual eggs, which are difficult to be macroscopically observed ${ }^{[52,53]}$. In particular, dermoscopy enables the visualization of the nits placed directly on the hair shaft and thus differentiates them from empty nits which commonly appear as translucent structures with a plane and fissured free end ${ }^{[34]}$. In this matter, dermoscopy can rapidly clarify any doubt by revealing the presence of lice and/or nits and differentiate from scales of different origin or pseudo-nits (hair casts, debris of hair spray or gel, or seborrheic scales), with subsequent remarkable effects on the therapeutic management. The advantage of dermoscopy is that it does not require hair removal and thus causes minimal discomfort to the patient. Furthermore, dermoscopy provides a useful diagnostic tool in children, where eyelashes are the most common site of infestation.

\section{Tungiasis}

Tungiasis is an ectoparasitic skin infection caused by the flea Tunga penetrans and is characterized by severe itch. The characteristic dermatoscopic pattern of tungiasis is a brown-to-black ring with a central pore, corresponding to the pigmented chitin surrounding the posterior portion of the parasite's body ${ }^{[54,55]}$. Additional dermatoscopic features include a "gray-blue blotch", indicating either the developing eggs within the abdomen of the parasite or the presence of hematin in its gastrointestinal 
tract ${ }^{[56,57]}$. Another dermoscopic finding is the "whitish chains" consisting of a number of whitish oval structures with a typical chain-like distribution corresponding to the parasite's eggs ${ }^{[58]}$.

\section{Cutaneous larva migrans}

Cutaneous larva migrans is a rare parasitic endemic disease most commonly found in tropical and subtropical countries, including South East Asia, and is caused by various hookworm species (helminths) such as Ancylostoma braziliense, Ancylostoma caninum or Uncinaria stenocephala. Although the clinical appearance is characterized by serpiginous erythema, following the movement of the larva under the skin dermoscopy can be useful for the diagnosis of cutaneous larva migrans. Dermoscopy reveals translucent brownish structureless areas in a segmental arrangement which corresponds to the body of the larva ${ }^{[59]}$.

\section{Cutaneous leishmaniasis}

Cutaneous leishmaniasis is a skin infection caused by any of the flagellate protozoans of the genus Leishmania and is transmitted to humans and animals by bloodsucking sand flies. Dermoscopically, the most common findings are characterized by diffuse erythema and vascular structures such as comma-like, linear or atypical, and arborizing vessels ${ }^{[60]}$. Early-stage lesions may have a yellow oval or tear-drop-shaped aspect known as yellow tears made from follicles due to the compression of follicular openings. In a later-stage of hyperkeratosis, hairpin and dotted vessels such as the so-called "white starburst"-like pattern due to central erosion and the peripheral hyperkeratosis can be seen ${ }^{[61]}$.

\section{Conclusion}

Dermoscopy represents a quick and easily applied, nontime-consuming, and non-invasive diagnostic tool, whose use and accessibility has been increasing in the last years. Dermoscopy is no more exclusively used for diagnosing skin tumors and it has gained ground in general dermatology. As it can be used not only by dermatologists but also by clinicians from other fields, it represents a useful diagnostic aid for the clinical diagnosis of many skin infections and infestations.

Our aim was to provide a comprehensive overview of the most typical examples of the current applications of dermoscopy in the daily clinical practice in the field of skin infections. Besides its role in diagnosis, dermoscopy is also useful for differential diagnosis, prognostic evaluation, and the monitoring of the response to treatment.

\section{Author contributions}

E Papakonstantinou and U Raap were involved in the overall concept and design of this review article. Acquisition, analysis and interpretation of data were performed by E Papakonstantinou. Critical revision of the manuscript's content was done by U Raap.

\section{Conflict of interest}

The authors declare no potential conflict of interest with respect to the research, authorship, and/or publication of this article.

\section{References}

1. Argenziano G, Soyer HP, Chimenti S, Talamini R, Corona $\mathrm{R}$, et al. Dermoscopy of pigmented skin lesions: Results of a consensus meeting via the Internet. J Am Acad Dermatol 2003; 48(5): 679-693. doi: 10.1067/mjd.2003.281.

2. Braun RP, Rabinovitz HS, Oliviero M, Kopf AW, Saurat JH. Dermoscopy of pigmented skin lesions. J Am Acad Dermatol 2005; 52(1): 109-121. doi: 10.1016/j.jaad. 2001.11.001.

3. Arrazola P, Mullani NA, Abramovits W. DermLite II: An innovative portable instrument for dermoscopy without the need of immersion fluids. Skinmed 2005; 4(2): 78-83. doi: 10.1111/j.1540-9740.2005.04363.x.

4. Hausermann P, Widmer A, Itin P. Dermatoscope as vector for transmissible diseases - No apparent risk of nosocomial infections in outpatients. Dermatology 2006; 212(1): 27-30. doi: 10.1159/000089018.

5. Kelly SC, Purcell SM. Prevention of nosocomial infection during dermoscopy? Dermatol Surg 2006; 32(4): 552-555. doi: 10.1111/j.1524-4725.2006.32112.x.

6. Zalaudek I, Argenziano G, Di Stefani A, Ferrara G, Marghoob AA, et al. Dermoscopy in general dermatology. Dermatology 2006; 212(1): 7-18. doi: 10.1159/000089015.

7. Micali G, Lacarrubba F, Musumeci ML, Massimino D, Nasca MR. Cutaneous vascular patterns in psoriasis. Int $\mathbf{J}$ Dermatol 2010; 49(3): 249-256. doi: 10.1111/j.13654632.2009.04287.x.

8. Lallas A, Kyrgidis A, Tzellos TG, Apalla Z, Karakyriou E, et al. Accuracy of dermoscopic criteria for the diagnosis of psoriasis, dermatitis, lichen planus and pityriasis rosea. Br J Dermatol 2012; 166(6): 1198-1205. doi: 10.1111/ j.1365-2133.2012.10868.x.

9. Vázquez-López F, Zaballos P, Fueyo-Casado A, Sánchez-Martín. A dermoscopy subpattern of plaque-type psoriasis: Red globular rings. Arch Dermatol 2007; 143(12): 1612. doi: 10.1001/archderm.143.12.1612. 
10. Micali G, Lacarrubba F, Massimino D, Schwarz RA. Dermoscopy: Alternative uses in daily clinical practice. J Am Acad Dermatol 2011; 64(6): 1135-1146. doi: 10.1016/j.jaad.2010.03.010.

11. Pan Y, Chamberlain AJ, Bailey M, Chong AH, Haskett M, et al. Dermoscopy aids in the diagnosis of the solitary red scaly patch or plaque-features distinguishing superficial basal cell carcinoma, intraepidermal carcinoma, and psoriasis. J Am Acad Dermatol 2008; 59(2): 268-274. doi: 10.1016/j.jaad.2008.05.013.

12. Zalaudek I, Giacomel J, Schmid K, Bondino S, Rosendahl C, et al. Dermoscopy of facial actinic keratosis, intraepidermal carcinoma, and invasive squamous cell carcinoma: A progression model. J Am Acad Dermatol 2012; 66(4): 589-597. doi: 10.1016/j.jaad.2011.02.011.

13. Giacomel J, Zalaudek I. Dermoscopy of superficial basal cell carcinoma. Dermatol Surg 2005; 31(12): 17101713. doi: 10.2310/6350.2005.31314.

14. Zalaudek I, Argenziano G. Dermoscopy subpatterns of inflammatory skin disorders. Arch Dermatol 2006; 142(6): 808. doi: 10.1001/archderm.142.6.808.

15. Vázquez-López F, Vidal AM, Zalaudek I. Dermoscopic subpatterns of ashy dermatosis related to lichen planus. Arch Dermatol 2010; 146(1): 110. doi: 10.1001/archdermatol.2009.300.

16. Vázquez-López F, Alvarez-Cuesta C, Hidalgo-García Y, Pérez-Oliva N. The handheld dermatoscope improves the recognition of Wickham striae and capillaries in Lichen planus lesions. Arch Dermatol 2001; 137(10): 1376.

17. Vázquez-López F, Gómez-Díez S, Sánchez J, Pérez-Oliva N. Dermoscopy of active lichen planus. Arch Dermatol 2007; 143(8): 1092. doi: 10.1001/archderm.143.8.1092.

18. Vázquez-López F, Manjón-Haces JA, Maldonado-Seral C, Raya-Aguado C, Pérez-Oliva N, et al. Dermoscopic features of plaque psoriasis and lichen planus: New observations. Dermatology 2003; 207(2): 151-156. doi: 10.1159/ 000071785.

19. Vazquez-Lopez F, Palacios-Garcia L, Gomez-Diez S, Argenziano G. Dermoscopy for discriminating between lichenoid sarcoidosis and lichen planus. Arch Dermatol 2011; 147(9): 1130. doi: 10.1001/archdermatol.2011.278.

20. Vázquez-López F, Maldonado-Seral C, López-Escobar M, Pérez-Oliva N. Dermoscopy of pigmented lichen planus lesions. Clin Exp Dermatol 2003; 28(5): 554-555. doi: 10.1046/j.1365-2230.2003.01302.x.

21. Chuh AAT. Collarette scaling in pityriasis rosea demonstrated by digital epiluminescence dermoscopy. Australas J Dermatol 2001; 42(4): 288-290. doi: 10.1046/j.14400960.2001.00538.x.

22. Lallas A, Argenziano G, Apalla Z, Gourhant JY, Zaballos $\mathrm{P}$, et al. Dermoscopic patterns of common facial inflammatory skin diseases. J Eur Acad Dermatol Venereol 2014; 28(5): 609-614. doi: 10.1111/jdv.12146.
23. Errichetti E, Stinco G. Dermoscopy in differential diagnosis of palmar psoriasis and chronic hand eczema. J Dermatol 2016; 43(4): 423-425. doi: 10.1111/13468138.13142.

24. Errichetti E, Stinco G. Th26e practical usefulness of dermoscopy in general dermatology. G Ital Dermatol Venereol 2015; 150(5): 533-546.

25. Navarini AA, Feldmeyer L, Töndury B, Fritsche P, Kamarashev J, et al. The yellow clod sign. Arch Dermatol 2011; 147(11): 1350. doi: 10.1001/archedrmatol.2011. 297.

26. Errichetti E, Piccirillo A, Stinco G. Dermoscopy as an auxiliary tool in the differentiation of the main types of erythroderma due to dermatological disorders. Int J Dermatol 2016; 55(12): e616-e618. doi: 10.1111/ijd.13322.

27. Lallas A, Apalla Z, Lefaki I, Tzellos T, Karatolias A, et al. Dermoscopy of early stage mycosis fungoides. J Eur Acad Dermatol Venereol 2013; 27(5): 617-621. doi: 10.1111/j.1468-3083.2012.04499.x.

28. Vázquez-López F, Marghoob AA. Dermoscopic assessment of long-term topical therapies with potent steroids in chronic psoriasis. J Am Acad Dermatol 2004; 51(5): 811-813. doi: 10.1016/j.jaad.2004.05.020.

29. Stinco G, Lautieri S, Valent F, Patrone P. Cutaneous vascular alterations in psoriatic patients treated with cyclosporine. Acta Derm Venereol 2007; 87(2): 152-154. doi: 10.2340/00015555-0216.

30. Stinco G, Buligan C, Maione V, Valent F, Patrone P. Videocapillaroscopic findings in the microcirculation of the psoriatic plaque during etanercept therapy. Clin Exp Dermatol 2013; 38(6): 633-637. doi: 10.1111/ced.12036.

31. Vázquez-López F, Kreusch J, Marghoob AA. Dermoscopic semiology: Further insights into vascular features by screening a large spectrum of nontumoral skin lesions. Br J Dermatol 2004; 150(2): 226-231. doi: 10.1111/j.1365-2133.2004.05753.x.

32. Teoli M, Di Stefani A, Botti E, Mio G, Chimenti S. Dermoscopy for treatment monitoring of viral warts. Dermatology 2006; 212: 318.

33. Bristow IR, Bowling J. Dermoscopy as a technique for the early identification of foot melanoma. J Foot Ankle Res 2009; 2: 14. doi: 10.1186/1757-1146-2-14.

34. Zalaudek I, Giacomel J, Cabo H, Di Stefani A, Ferrara G, et al. Entodermoscopy: A new tool for diagnosing skin infections and infestations. Dermatology 2008; 16(1): 14-23. doi: 10.1159/000109353.

35. Watanabe T, Yoshida Y, Yamamoto O. Differential diagnosis of pearly penile papules and penile condyloma acuminatum by dermoscopy. Eur J Dermatol 2010; 20(3): 414-415. doi: 10.1684/ejd.2010.0944.

36. Dong H, Shu D, Campbell TM, Frühauf J, Soyer P, et al. Dermoscopy of genital warts. J Am Acad Dermatol 2011; 64(5): 859-864. doi: 10.1016/j.jaad.2010.03.028. 
37. Veasev JV, de Souza Framil VM, Nadal SR, Marta AC, Lellis RF. Genital warts: Comparing clinical findings to dermatoscopic aspects, in vivo reflectance confocal features and histopathologic exam. An Bras Dermatol 2014; 89(1): 137-140. doi: 10.1590/abd1806-4841.20141917.

38. Morales A, Puig S, Malvehy J, Zaballos P. Dermoscopy of molluscum contagiosum. Arch Dermatol 2005; 141(12): 1644. doi: 10.1001/archderm.141.12.1644.

39. Ianhez M, Cestari SdaCP, Enokihara MY, Seize MBdPM. Dermoscopic patterns of molluscum contagiosum: A study of 211 lesions confirmed by histopathology. An Bras Dermatol 2011; 86(1): 74-79. doi: 10.1590/S036505962011000100009.

40. Haliasos EC, Kerner M, Jaimes-Lopez N, Rudnicka L, Zalaudek I, et al. Dermoscopy for the pediatric dermatologist part I: Dermoscopy of pediatric infectious and inflammatory skin lesions and hair disorders. Pediatr Dermatol 2013; 30(2): 163-171. doi: 10.1111/pde.12097.

41. Zaballos P, Ara M, Puig S, Malvehy J. Dermoscopy of molluscum contagiosum: A useful tool for clinical diagnosis in adulthood. J Eur Acad Dermatol Venereol 2006; 20(4): 482-483. doi: 10.1111/j.1468-3083.2006.01480.x.

42. Smith SB, Beals SL, Elston DM, Meffert JJ. Dermoscopy in the diagnosis of tinea nigra plantaris. Cutis 2001; 68(6): 377-380.

43. Piliouras P, Alison S, Rosendahl C, Buettner PG, Weedon D. Dermoscopy improves diagnosis of tinea nigra: A study of 50 cases. Australas J Dermatol 2011; 52(3): 191-194. doi: 10.1111/j.1440-0960.2011.00790.x.

44. Zhou H, Tang XH, De Han J, Chen MK. Dermoscopy as an ancillary tool for the diagnosis of pityriasis versicolor. J Am Acad Dermatol 2015; 73(6): e205-e206. doi: 10.1016/j.jaad.2015.08.058.

45. Neynaber S, Wolff H. Diagnosis of scabies with dermoscopy. CMAJ 2008; 178(12): 1540-1541. doi: 10.1503/cmaj.061753.

46. Dupuy A, Dehen L, Bourrat E, Lacroix C, Benderdouche M, et al. Accuracy of standard dermoscopy for diagnosing scabies. J Am Acad Dermatol 2007; 56(1): 53-62. doi: 10.1016/j.jaad.2006.07.025.

47. Argenziano G, Fabbrocini G, Delfino M. Epiluminescence microscopy: A new approach to in vivo detection of Sarcoptes scabiei. Arch Dermatol 1997; 133(6): 751-753. doi: 10.1001/archderm.1997.03890420091011.

48. Lacarrubba F, D'Amico V, Nasca MR, Dinotta F, Micali G. Use of dermatoscopy and videodermatoscopy in therapeutic follow-up: A review. Int J Dermatol 2010; 49(8): 866-873. doi: 10.1111/j.1365-4632.2010.04581.x.

49. Suh KS, Han SH, Lee KH, Park JB, Jung SM, et al. Mites and burrows are frequently found in nodular scabies by dermoscopy and histopathology. J Am Acad Dermatol 2014; 71(5): 1022-1023. doi: 10.1016/j.jaad.2014.06.028.

50. Chouela E, Abeldaño A, Pellerano G, Hernández MI. Diagnosis and treatment of scabies: A practical guide. Am J Clin Dermatol 2002; 3(1): 9-18. doi: 10.2165/ 00128071-200203010-00002.

51. Hamm H, Beiteke U, Höger PH, Seitz CS, Thaci D, et al. Treatment of scabies with 5\% permethrin cream: Results of a German multicenter study. J Dtsch Dermatol Ges 2006; 4(5): 407-413. doi: 10.1111/j.1610-0387.2006.05941.x.

52. Di Stefani A, Hofmann-Wellenhof R, Zalaudek I. Dermoscopy for diagnosis and treatment of pediculosis capitis. J Am Acad Dermatol 2006; 54(5): 909-911. doi: 10.1016/j.jaad.2005.11.1083.

53. Bakos RM, Bakos L. Dermoscopy for diagnosis of pediculosis capitis. J Am Acad Dermatol 2007; 57(4): 727-728. doi: 10.1016/j.jaad.2006.11.011.

54. Criado PR, Landman G, dos Reis VMS, Belda W Jr. Tungiasis under dermoscopy: In vivo and ex vivo examination of the cutaneous infestation due to Tunga penetrans. An Bras Dermatol 2013; 88(4): 649-651. doi: 10.1590/ abd1806-4841.20132071.

55. Bauer J, Forschner A, Garbe C, Rőcken M. Dermoscopy of tungiasis. Arch Dermatol 2004; 140(6): 761-763. doi: 10.1001/archderm.140.6.761.

56. Di Stefani A, Rudolph CM, Hofmann-Wellenhof R, Müllegger RR. An additional dermoscopic feature of tungiasis. Arch Dermatol 2005; 141(8): 1045-1046. doi: 10.1001/archderm.141.8.1045.

57. Bauer J, Forschner A, Garbe C, Rőcken M. Variability of dermoscopic features of tungiasis. Arch Dermatol 2005; 141(5): 643-644. doi: 10.1001/archderm.141.5.643.

58. Bakos RM, Bakos L. 'Whitish chains': A remarkable in vivo dermoscopic finding of tungiasis. B J Dermatol 2008; 159(4): 991-992. doi: 10.1111/j.1365-2133.2008.08782.x.

59. Veraldi S, Schianchi R, Carrera C. Epiluminescence microscopy in cutaneous larva migrans. Acta Derm Venereol 2000; 80(3): 233.

60. Llambrich A, Zamballos P, Terrasa F, Torne I, Puig S, et al. Dermoscopy of cutaneous leishmaniasis. Br J Dermatol 2009; 160(4): 756-761. doi: 10.1111/j.1365-2133. 2008.08986.x.

61. Buljan M, Zalaudek I, Massone C, Hofmann-Wellenhof R, Fink-Puches R, et al. Dermoscopy and reflectance confocal microscopy in cutaneous leishmaniasis on the face. Australas J Dermatol 2016; 57(4): 316-318. doi: 10.1111/ ajd.12404. 